\title{
Phantom cosmologies and fermions
}

\author{
Luis P. Chimento ${ }^{1}$, Fernando P. Devecchi ${ }^{2}$, Mónica Forte ${ }^{1}$ and Gilberto M. Kremer ${ }^{2}$ \\ 1 Dpto. de Física, Facultad de Ciencias Exactas y Naturales, \\ Universidad de Buenos Aires, Ciudad Universitaria \\ Pabellón I, 1428 Buenos Aires, Argentina \\ chimento@df.uba.ar, monicaforte@fibertel.com.ar \\ 2 Depto. de Física, Universidade Federal do Paraná \\ Caixa Postal 19044, 81531-990 Curitiba, Brazil \\ devecchi@fisica.ufpr.br, kremer@fisica.ufpr.br
}

(Dated: July 30, 2007)

\begin{abstract}
Form invariance transformations can be used for constructing phantom cosmologies starting with conventional cosmological models. In this work we reconsider the scalar field case and extend the discussion to fermionic fields, where the "phantomization" process exhibits a new class of possible accelerated regimes.
\end{abstract}

\section{INTRODUCTION}

The issue of form invariance symmetries [1] has been invoked recently to extract different evolution regimes from Friedmann-Robertson-Walker (FRW) and Bianchi type $\mathrm{V}$ cosmologies [2]. This is the case when we obtain the so-called phantom cosmologies [3]- 6] from ordinary regimes like universes filled with barotropic fluids [7]. These phantom models generate solutions that contain negative pressure situations, therefore promoting positive accelerated expansions [8], 9]. The case were the gravitational source is a bosonic field was investigated in [1], [3], [5], and the question that follows would be what happens when the field promoting the universe expansion is a fermion whose dynamics is described by Dirac equations. These kind of models has been analyzed in several works [10], [11] where it was shown that regimes with ordinary matter/dark energy transitions are possible solutions of Einstein and Dirac equations. Taking into account these ideas, the main point of discussion of this work is to investigate the form invariance transformations (FIT) when the Klein-Gordon dynamics is replaced by the Dirac equations in a FRW space-time. The manuscript is structured as follows: in section II we review the basic ideas behind the FIT in cosmology; in section III we focus on the scalar field case. In section IV we analyze the symmetries behind the fermionic formulations. In section $\mathrm{V}$ we introduce the dual transformation and the associated "phantomization" process. Finally in section VI we display our conclusions.

\section{INTERNAL SYMMETRY IN FRW}

An interesting method to obtain new exact solutions for the Einstein equations from already existing ones, by using the FIT, has been shown in a series of papers [1]-[6], [12]-14.

In spatially flat perfect fluid FRW cosmologies, such transformations can be viewed as a prescription relating the quantities $a, H, \rho$ and $p$ in a given initial scenario to quantities $\bar{a}, \bar{H}, \bar{\rho}$ and $\bar{p}$ corresponding to a new cosmological model.

As a starting point we present a quick review of this internal symmetry with the purpose of investigating it in bosonic and fermionic cases. As is well-known, the Einstein equations for a flat FRW cosmological model with scale factor $a$, and filled with a perfect fluid with energy density $\rho$ and pressure $p$, are

$$
\begin{aligned}
& 3 H^{2}=\rho, \\
& \dot{\rho}+3 H(\rho+p)=0,
\end{aligned}
$$

where $H=\dot{a} / a$. The correspondent FIT is given by [1]

$$
\begin{aligned}
& \bar{\rho}=\bar{\rho}(\rho), \\
& \bar{H}=\left(\frac{\bar{\rho}}{\rho}\right)^{1 / 2} H, \\
& \bar{p}=-\bar{\rho}+\left(\frac{\rho}{\bar{\rho}}\right)^{1 / 2}(\rho+p) \frac{d \bar{\rho}}{d \rho},
\end{aligned}
$$

where $\bar{\rho}=\bar{\rho}(\rho)$ is an invertible arbitrary function and the set of transformations (3)-(5) give rise to the form invariance symmetry (FIS) group . For the perfect fluid case, $p=(\gamma-1) \rho$, the barotropic indexes of the original and transformed fluid are related by

$$
\bar{\gamma}=\left(\frac{\rho}{\bar{\rho}}\right)^{3 / 2} \frac{d \bar{\rho}}{d \rho} \gamma .
$$

For later application it will useful to investigate the transformation rule for the quantities which characterize the geometry and the fluid when the transformation is generated by [1]

$$
\bar{\rho}=n^{2} \rho
$$

where $n$ is the constant parameter of the FIS group. Hence, for the geometrical quantities we get

$$
\bar{H}=n H, \quad \rightarrow \quad \bar{a}=a^{n},
$$


and

$$
\begin{gathered}
\bar{w}=\bar{\gamma}-1=\frac{\gamma}{n}-1, \\
\bar{p}=\bar{w} \bar{\rho}=\left(\frac{\gamma}{n}-1\right) \bar{\rho},
\end{gathered}
$$

for the state parameter $w$ and the pressure of the fluid.

Let us analyze the equation of state (10) generated by transformation (7) when the seed fluid represents a normal fluid with $\gamma>0$. We can infer: (i) when $0<$ $n<\gamma$ it follows that $\bar{w}>0$ and the cosmological fluid behaves as a normal fluid; (ii) when $n>\gamma$ it follows that $-1<\bar{w}<0$ and the cosmological fluid behaves as a quintessence constituent; (iii) when $n<0$ it follows that $\bar{w}<-1$ and the cosmological fluid behaves as a phantom constituent.

\section{THE BOSONIC CASE}

In this section we analyze the transformation rules for a scalar field $\phi$, self-interacting through a potential $V(\phi)$, under the simple transformation generated by (7) [1]. Applying Eqs. (44) and (5) on the dynamical quantities associated with the scalar field, i.e., its energy density $\rho_{\phi}=\dot{\phi}^{2} / 2+V(\phi)$ and pressure $p_{\phi}=\dot{\phi}^{2} / 2-V(\phi)$, we obtain

$$
\begin{aligned}
& \dot{\bar{\phi}}^{2}=n \dot{\phi}^{2}, \\
& \bar{V}(\bar{\phi})=n(n-1) \frac{\dot{\phi}^{2}}{2}+n^{2} V(\phi) .
\end{aligned}
$$

In order to study the action of the group, below, we will generate the set of power law solution starting for a particular seed one.

\section{A. Bosonic power law group}

Let us solve the Einstein-Klein-Gordon equations for a free scalar field, $V=0$. The solutions of those equations are given by

$$
a^{( \pm)}=( \pm t)^{1 / 3}, \quad \phi= \pm \sqrt{\frac{2}{3}} \ln |t|, \quad V=0 .
$$

where the branches $a^{(+)}$and $a^{(-)}$are defined for $t>0$ and $t<0$ respectively. Now, we use the latter as a seed set for the FIS group. To do that first we calculate the transformed quantities when the group is acting on the above solutions

$$
\begin{aligned}
& \dot{\bar{\phi}}^{2}=n \dot{\phi}^{2}, \\
& \bar{V}(\bar{\phi})=n(n-1) \frac{\dot{\phi}^{2}}{2} .
\end{aligned}
$$

Finally combining the seed set (13) with the above equations we obtain the power law solutions together with the scalar fields and corresponding potentials

$$
\begin{aligned}
& \bar{a}^{( \pm)}=( \pm t)^{n / 3}, \\
& \bar{\phi}= \pm \sqrt{\frac{2 n}{3}} \ln |t|, \\
& \bar{V}(\bar{\phi})=\frac{n}{3}(n-1) e^{\mp \sqrt{6 / n} \bar{\phi}} .
\end{aligned}
$$

For the identical transformation, $n=1$, the latter equations (16)-(18) reduce to the seed solution (13). We conclude that power law solutions can be generated starting from that, corresponding to the free scalar field.

\section{THE FERMIONIC CASE}

In this section we extend the FIT to a fermionic field satisfying the Dirac equation in curved space time. As required by equations (1) and (2) we must calculate the energy density and the hydrostatic pressure of the fermionic field. We have 11]

$$
\begin{gathered}
\rho_{\psi}=m(\bar{\psi} \psi)+V, \\
p_{\psi}=\frac{d V}{d \psi} \frac{\psi}{2}+\frac{\bar{\psi}}{2} \frac{d V}{d \bar{\psi}}-V,
\end{gathered}
$$

where $\psi$ and $\bar{\psi}=\psi^{\dagger} \gamma^{0}$ are the spinor field and its adjoint, respectively.

To obtain the transformation properties of $\rho_{\psi}$ and $p_{\psi}$ we express the potential in terms of the scalar invariant $X=(\bar{\psi} \psi)^{2}$ and the pseudo-scalar invariant $Y=$ $\left(i \bar{\psi} \gamma^{5} \psi\right)^{2}$. To this end, we suppose a generic potential $V=V(X, Y)$, that include, among others, the NambuJona-Lasinio potential [11]. For this kind of potential the hydrostatic pressure becomes

$$
p_{\psi}=2 X \frac{\partial V}{\partial X}+2 Y \frac{\partial V}{\partial Y}-V .
$$

Applying the transformation rules (3)-(15), (7) to the energy density (19) and the hydrostatic pressure (21) we find that

$$
\begin{gathered}
\bar{m} \sqrt{\bar{X}}+\bar{V}=n^{2}(m \sqrt{X}+V), \\
2 \bar{X} \frac{\partial \bar{V}}{\partial \bar{X}}+2 \bar{Y} \frac{\partial \bar{V}}{\partial \bar{Y}}+\bar{m} \sqrt{\bar{X}} \\
=n\left[2 X \frac{\partial V}{\partial X}+2 Y \frac{\partial V}{\partial Y}+m \sqrt{X}\right] .
\end{gathered}
$$

Inserting $\bar{V}$, from Eq.(22), into Eq.(23) we obtain the following equation

$$
\left(\frac{m}{2 \sqrt{X}}+\frac{\partial V}{\partial X}\right)\left[\bar{X} \frac{\partial X}{\partial \bar{X}}+\bar{Y} \frac{\partial X}{\partial \bar{Y}}-\frac{X}{n}\right]
$$




$$
+\frac{\partial V}{\partial Y}\left[\bar{X} \frac{\partial Y}{\partial \bar{X}}+\bar{Y} \frac{\partial Y}{\partial \bar{Y}}-\frac{Y}{n}\right]=0 .
$$

All transformations satisfying the last condition (24) represent internal symmetries of the Einstein equations with a fermionic source, provide that condition becomes an identity for any potential and particle mass. Then the two square brackets must vanish

$$
\begin{aligned}
& \bar{X} \frac{\partial X}{\partial \bar{X}}+\bar{Y} \frac{\partial X}{\partial \bar{Y}}=\frac{X}{n} \\
& \bar{X} \frac{\partial Y}{\partial \bar{X}}+\bar{Y} \frac{\partial Y}{\partial \bar{Y}}=\frac{Y}{n} .
\end{aligned}
$$

In addition, by imposing the condition

$$
\bar{m}(n=1, \bar{X}=X, \bar{V}=V)=m,
$$

on the transformed fermionic mass, the Eq. (22) becomes an identity for the identity transformation $n=1, \bar{X}=X$ and $\bar{V}=V$. Hence, any solution of the coupled system of partial differential equations (25) and (26), satisfying the condition (27), is a FIS transformation of the EinsteinDirac dynamic.

For the general massive fermionic field whose potential depends only on $X$, the solution of (25) along with (22) lead to the general FIT

$$
\begin{aligned}
& \bar{X}=X^{n}, \\
& \bar{V}=n^{2} V+n^{2} m\left(\sqrt{X}-X^{n / 2}\right), \\
& \bar{m}=n^{2} m .
\end{aligned}
$$

Also, from Eqs. (21), (19) and (21) we get $X \propto a^{-6}$ and $\bar{a}=a^{n}$.

\section{A. Fermionic power law group}

Following the same methodology as the bosonic scalar field, let us investigate the two simple cases, (i) the free massive fermionic particle and (ii) the massless fermionic particle with a polynomial potential.

In the free case, the energy density (19) and pressure (21) of the fermionic particle are given by

$$
\rho=m \sqrt{X}, \quad p=0, \quad V=0 .
$$

Solving the Einstein equations we obtain

$$
a^{( \pm)}=a_{0}( \pm t)^{2 / 3}, \quad X=\frac{16}{9 m^{2}}\left(\frac{a_{0}}{a}\right)^{6},
$$

where $a_{0}$ is an integration constant. Suppose now that we are interested in comparing this cosmological model with other one driven by a potential, which is assumed to be dependent only on $X$. This can be done by inserting the seed configuration (31)-(32) into the transformations (5), (7), (8) and (28)-(30). Then, we find the full set of power law solutions along with the potential, energy density and pressure characterizing the transformed fermionic configuration:

$$
\begin{gathered}
\bar{a}^{( \pm)}=\bar{a}_{0}( \pm t)^{2 n / 3}, \quad \bar{a}_{0}=a_{0}^{n}, \\
\bar{X}=\left(\frac{16}{9 m^{2}}\right)^{n}\left(\frac{\bar{a}_{0}}{\bar{a}}\right)^{6}, \\
\bar{V}=\bar{m}\left(\bar{X}^{1 / 2 n}-\bar{X}^{1 / 2}\right), \quad \bar{m}=n^{2} m, \\
\bar{\rho}=\bar{m} \bar{X}^{1 / 2 n}, \quad \bar{p}=\left(\frac{1}{n}-1\right) \bar{\rho} .
\end{gathered}
$$

The Dirac equations in a FRW background for the seed and transformed configurations read

$$
\begin{aligned}
& \dot{\psi}+\frac{3}{2} H \psi+\imath m \gamma^{0} \psi+\imath \gamma^{0} \frac{d V}{d \bar{\psi}}=0, \\
& \dot{\bar{\psi}}+\frac{3}{2} \bar{H} \bar{\psi}+\imath \bar{m} \gamma^{0} \bar{\psi}+\imath \gamma^{0} \frac{d \bar{V}}{d \overline{\bar{\psi}}}=0,
\end{aligned}
$$

along with the corresponding equation for the adjoint $\bar{\psi}$ and $\bar{\psi}$. In order to preserve the own characteristics of the fermionic particles, we assume that the Dirac matrices remain invariants under a FIS transformation.

Solving the Dirac equation (38) for the new configuration (33)-(36), we obtain the spinor field solution

$$
\bar{\psi}(t)=\frac{1}{t^{n}}\left(\begin{array}{c}
\bar{b}_{1} e^{-\imath \bar{m} \tau_{n}} \\
\bar{b}_{2} e^{-\imath \bar{m} \tau_{n}} \\
\bar{d}_{1}^{*} e^{\imath \bar{m} \tau_{n}} \\
\bar{d}_{2}^{*} e^{\imath \bar{m} \tau_{n}}
\end{array}\right),
$$

where

$$
\left|\bar{b}_{1}\right|^{2}+\left|\bar{b}_{2}\right|^{2}-\left|\bar{d}_{1}\right|^{2}-\left|\bar{d}_{2}\right|^{2}=\left(\frac{4 n^{2}}{3 \bar{m}}\right)^{n}>0,
$$

and

$$
\tau_{n}=\frac{1}{n}\left(\frac{4 n^{2}}{3 \bar{m}}\right)^{1-n} \int t^{2(n-1)} d t .
$$

For $n=1$ the latter becomes the spinor field corresponding to the seed solution (31)-(32). In addition, the Einstein equations for the new configuration are

$$
3 \bar{H}^{2}=\bar{\rho}, \quad-2 \dot{\bar{H}}=\bar{\rho}+\bar{p},
$$

so, the new solution (33)-(36) can be associated with a fermionic barotropic fluid whose equation of state is $\bar{\gamma}=$ $1+\bar{p} / \bar{\rho}=1 / n$. When $n=1$, identical transformation, the solution (33)-(36) become the seed solution (31)-(32), which can be identified with some kind of fermionic dust matter.

Now, we consider the massless case and investigate a spinor field driven by a potential $V=V(X)$ depending on $X$. Here, the energy density $\rho$ and the pressure $p$ associated with the spinor field are given by:

$$
\rho=V, \quad p=2 X \frac{d V}{d X}-V .
$$


The imposition of the energy density transformation $\bar{\rho}=$ $n^{2} \rho$ reduces the transformation rules (28)- (30) to

$$
\bar{X}=X^{n}, \quad \bar{V}=n^{2} V, \quad \bar{m}=0 .
$$

By choosing the seed potential $V=\sqrt{X}$ we obtain

$$
\rho=\sqrt{X}, \quad p=0,
$$

and from the Einstein equations it follows

$$
a^{( \pm)}=a_{0}( \pm t)^{2 / 3}, \quad X=\frac{16}{9}\left(\frac{a_{0}}{a}\right)^{6} .
$$

Actually, from the mathematical point of view, this solution and the corresponding spinor field may be obtained from the above seed solution (31)-(32) and the general spinor field (39)-(41) respectively by making $m=1$. However, both cases, $m \neq 0$ and $m=0$, are absolutely different because a FIT that relates them does not exist, as can be seen from Eq. (27). In fact, for the $m=0$ case, the solution for the transformed fermionic configuration read

$$
\begin{gathered}
\bar{a}^{( \pm)}=\bar{a}_{0}( \pm t)^{2 n / 3}, \quad \bar{a}_{0}=a_{0}^{n}, \\
\bar{X}=\left(\frac{16}{9}\right)^{n}\left(\frac{\bar{a}_{0}}{\bar{a}}\right)^{6}, \\
\bar{V}=n^{2} \bar{X}^{1 / 2 n}, \\
\bar{\rho}=n^{2} \bar{X}^{1 / 2 n}, \quad \bar{p}=\left(\frac{1}{n}-1\right) \bar{\rho} .
\end{gathered}
$$

Thus, comparing the transformed configurations (33)(36) with (47)-(50) for $m=1$, we see that both potential are different and the dynamics too.

\section{DUALITY AND PHANTOM COSMOLOGIES}

The "phantomization" process means that the energy density of the expanding cosmological model under analysis must satisfy $\dot{\rho}>0$ or equivalently the weak energy condition (WEC), $(\rho+p)<0$, must be violated. In a FRW background the previous conditions become $H>0$ and $\dot{H}>0$. Therefore, one can infer two cases, namely, (a) $\rho$ has an asymptote $\rho \rightarrow \Lambda$ for $t \rightarrow \infty$, or (b) $\rho$ grows without limit. In the first case the scale factor tends to a de Sitter solution $a \rightarrow \exp \sqrt{\Lambda / 3} t$. For the second case by supposing the following asymptotic behavior for the energy density $\rho \rightarrow \rho_{0} a^{k}$ with $k>0$, the asymptotic solution of Friedmann equation reads

$$
\begin{aligned}
& a^{(-)} \rightarrow\left[\frac{2 \sqrt{3}}{k \sqrt{\rho_{0}}\left(t_{0}-t\right)}\right]^{2 / k}, \quad t<t_{0}, \\
& a^{(+)} \rightarrow\left[\frac{2 \sqrt{3}}{k \sqrt{\rho_{0}}\left(t-t_{0}\right)}\right]^{2 / k}, \quad t>t_{0} .
\end{aligned}
$$

The expanding solution $a^{(-)}$is defined for $t<t_{0}$ and ends in a big rip at $t=t_{0}$, since the scale factor diverges at the finite time $t_{0}$ resulting into a singularity in the future. Unlike the former, the contracting solution $a^{(+)}$ begins from a singularity in the past at $t=t_{0}$. To sum up, $a^{(-)}$is the "phantomization" of the solution $1 / a^{(-)}$ which ends in a big crunch at $t=t_{0}$. In terms of FIS the "phantomization" process means that there is a duality between two solutions of the Friedmann equation, i.e., between $1 / a^{(-)}$and $a^{(-)}$[12]-[16]. In fact, the class of FIT generated by $n=-1$ :

$$
\rho \rightarrow \bar{\rho}=\rho, \quad p+\rho \rightarrow \bar{p}+\bar{\rho}=-(p+\rho),
$$

with

$$
H \rightarrow \bar{H}=-H, \quad a \rightarrow \bar{a}=1 / a,
$$

transforms a contracting scale factor, $H<0$, satisfying the WEC, $\rho+p>0$ into an expanding one, $\bar{H}>0$, which violates the WEC, $\bar{\rho}+\bar{p}<0$. The latter, dubbed dual transformation, is crucial because of their applicability as a method of transforming a conventional cosmological model into a phantom one by performing a FIT.

In the bosonic case the expressions (11) and (12) become

$$
\begin{aligned}
& \dot{\bar{\phi}}^{2}=-\dot{\phi}^{2}, \\
& \bar{V}(\bar{\phi})=\dot{\phi}^{2}+V(\phi) .
\end{aligned}
$$

and this implies into $\bar{\phi}=i \phi$. That is, the transformed scalar field is related to the original one by a Wick rotation, and we finally have

$$
\bar{\rho}=\frac{1}{2} \dot{\bar{\phi}}^{2}+\bar{V}(\bar{\phi})
$$

The sign in the kinetic part of the energy density, $\dot{\bar{\phi}}^{2}=$ $-\dot{\phi}^{2}<0$, indicates that what we actually have now is a phantom cosmology, with a phantom field $\bar{\phi}$, driven by the potential $\bar{V}(\bar{\phi})$, which is a real function of $\phi$. Finally we see that the relationship $\bar{H}=-H$, gives in turn for the scale factor the transformation law $\bar{a} \propto a^{-1}$.

For the fermionic matter, in the case of having a potential depending of $X$, the "phantomization" induces the following transformation rules for the variables $X$ and $V$

$$
\begin{aligned}
& \bar{X}=\frac{1}{X} \\
& \bar{V}=m \sqrt{X}\left(1-\frac{1}{X}\right)+V .
\end{aligned}
$$

and

$$
\bar{\gamma}=-\gamma
$$

for the barotropic index of the spinor field. Hence, there is a duality between a contracting universe filled with an ordinary spinor field, $\gamma>0$, and an expanding universe filled with a phantom spinor field $\bar{\gamma}<0$. 


\section{CONCLUSION}

We have found the symmetry transformations under which the Einstein equations, for a spatially flat FRW space-time filled with bosonic and spinor fields, preserve their form. This group of transformation has been used to obtain power-law solutions from a seed one, for bosonic and fermionic fluids. We have given special attention to the $n=-1$ case, which express the duality between non-accelerated and accelerated scenarios and viceversa. Thus, starting from a contracting spatially flat FRW cosmological models we get, after using the dual transformation, a super-accelerated spatially flat FRW cosmological models, i.e., the "phantomization" of the model. We have shown that bosonic and fermionic fields behave differently under a dual transformation, the former be- comes imaginary, whereas the latter changes the sign of the phase and inverts its asymptotic limits.

\section{Acknowledgments}

The authors acknowledge the partial support under project 24/07 of the agreement SECYT (Argentina) and CAPES 117/07 (Brazil). LPC thanks the University of Buenos Aires for partial support under project X224, and the Consejo Nacional de Investigaciones Científicas y Técnicas under project 5169. FPD and GMK acknowledge the support by Conselho Nacional de Desenvolvimento Científico e Tecnológico (CNPq).
[1] L. P. Chimento, Phys. Rev. D 65 (2002) 063517.

[2] J. M. Aguirregabiria, L. P. Chimento, A. S. Jakubi and R. Lazkoz, Phys. Rev. D 67, 083518 (2003).

[3] L. P. Chimento and R. Lazkoz, Phys. Rev. Lett. 91, 211301 (2003).

[4] J. M. Aguirregabiria, L. P. Chimento and R. Lazkoz, Phys. Rev. D 70, 023509 (2004)

[5] L. P. Chimento and R. Lazkoz, Int. J. Mod. Phys. D 14, 587 (2005).

[6] L. P. Chimento, Phys. Lett. B 633, 9 (2006).

[7] R. R. Caldwell, Phys. Lett. B 545, 23 (2002); R. R. Caldwell, M. Kamionkowski and N. N. Weinberg, Phys. Rev. Lett. 91, 071301 (2003); P. F. Gonzalez-Diaz, Phys. Rev. D 68, 021303 (2003).

[8] V. B. Johri, Phys. Rev. D 70, 041303 (2004); P. Singh, M. Sami and N. Dadhich, Phys. Rev. D 68, 023522 (2003); X. z. Li and J. g. Hao, Phys. Rev. D 69, 107303 (2004).

[9] M. P. Dabrowski, T. Stachowiak and M. Szydlowski, Phys. Rev. D 68, 103519 (2003).

[10] Y. N. Obukhov, Phys. Lett. A 182, 214 (1993)
arXiv:gr-qc/0008015; B. Saha and G. N. Shikin, Gen. Rel. Grav. 29, 1099 (1997) arXiv:gr-qc/9609056; B. Saha, Phys. Rev. D 64, 123501 (2001) arXiv:gr-qc/0107013; C. Armendariz-Picon and P. B. Greene, Gen. Rel. Grav. 35, 1637 (2003) arXiv:hep-th/0301129; B. Saha and T. Boyadjiev, Phys. Rev. D 69, 124010 (2004) arXiv:gr-qc/0311045; B. Saha, Phys. Rev. D 74, 124030 (2006);

[11] M. O. Ribas, F. P. Devecchi and G. M. Kremer, Phys. Rev. D 72, 123502 (2005) arXiv:gr-qc/0511099.

[12] M. Cataldo and L. P. Chimento, arXiv:gr-qc/0506090

[13] L. P. Chimento and D. Pavon, Phys. Rev. D 73, 063511 (2006).

[14] L. P. Chimento and W. Zimdahl, arXiv:gr-qc/0609104

[15] Y. f. Cai, H. Li, Y. S. Piao and X. m. Zhang, Phys. Lett. B 646, 141 (2007); G. Veneziano, Phys. Lett. B 265, 287 (1991).

[16] L. P. Chimento and R. Lazkoz, Class. Quant. Grav. 23, 3195 (2006) 